\title{
Review
}

\section{Friction and Adhesion of Polysaccharides}

\author{
Rowena Crockett \\ Swiss Federal laboratories for Materials Science and Technology, Empa \\ Ueberlandstrasse 129, CH 8600 Duebendorf, Switzerland \\ Corresponding author: rowena.crockett@empa.ch
}

( Manuscript received 30 April 2014; accepted 25 August 2014; published 15 October 2014 )

(Presented at the International Tribology Forum Tokyo 2013 at the Katsushika Campus, Tokyo University of Science, October 2013)

\begin{abstract}
This paper provides a brief overview of investigations that have been carried out on the tribological properties of polysaccharides. Much of the work into long chain carbohydrates focuses on adhesion due to the propensity of these molecules to form hydrogen bonds. Polysaccharides play an important role in bioadhesion, but are also used in the mining industry to assist in the separation of minerals. Despite the high adhesion associated with polysaccharides, investigations showing that they can be used to achieve low friction have also been reported.In order to observe an effect on the friction between sliding surfaces in the boundary regime, the polysaccharide must first adsorb onto the surface. Although hydrogen bonding is the dominant interaction for polysaccharides, they can also interact through hydrophobic interactions in aqueous solution, providing the structure of the carbohydrate allows this. Many polysaccharides are charged and can, therefore, be adsorbed onto charged surfaces via electrostatic interactions. If the sliding contact is symmetric, that is both surfaces are of the same material, then it could be expected that bridging between the surfaces would occur due to the same interactions being formed on both sides. However, friction studies have been carried out that show that this phenomenon does not necessarily lead to high friction coefficients.
\end{abstract}

Keywords: polysaccharides, carbohydrates, adhesion, friction, hydrogen bonding

\section{Introduction}

Carbohydrates are often associated not only with low friction in natural systems but also with high adhesion [1-3]. These can be in the form of oligosaccharide chains attached to protein backbones to form mucins or large polysaccharide chains. Polysaccharides are long polymers whereas oligosaccharides are shorter chains of monosaccharide, however, the difference is not clearly defined. Much of the research into the lubricating properties of carbohydrates is motivated by an interest in understanding the phenomenon involved and in mimicking the natural properties in synthetic systems. Additionally, polysaccharides derived from natural systems are often investigated as potential aqueous based lubricants [4-7]. For example, a sulfated polysaccharide from a red microalga Porphyridium sp. has been shown to have excellent lubricating properties on the macro and micro scales [5-7]. However, the low friction observed when polysaccharides lubricate sliding surfaces is not always desirable. The loss of adhesion between train wheels and track has been attributed in part to the polysaccharide pectin in the leaves that fall onto the train line [8].

The contradictory properties of low friction and high adhesion are mirrored in the differing tribological behavior of polysaccharides in laboratory experiments. For example, the dependence of the friction forces on water content in polysaccharides gels, when measured in water, is dependent on the type of polysaccharide. For agarose and gellan gels, the friction force increases with increasing water concentration, whereas for $\mathrm{K}$-carrageenan gels, the friction decreases with increasing water content (Fig. 1) [9]. Both gellan gum and $\mathrm{K}$-carrageenan gels show a negative dependence of friction on applied pressure after some critical load when measured in air. Agarose gel does not show this behavior. These differences in the behavior of the polysaccharides were attributed to the different interactions with the glass substrate used as the counter-face. Thus, where there were attractive forces between polysaccharide and glass, as in the case of agarose, the friction force was higher and showed a weak dependence on load. On the other hand, gellan and $\mathrm{K}$-carrageenan have repulsive interactions with the glass surface and give low friction forces that show a strong load dependence [9]. 


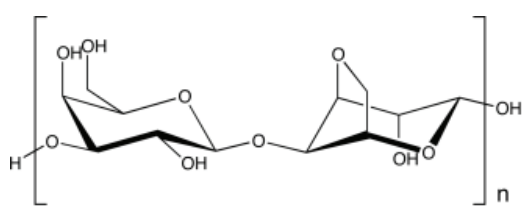

(a)

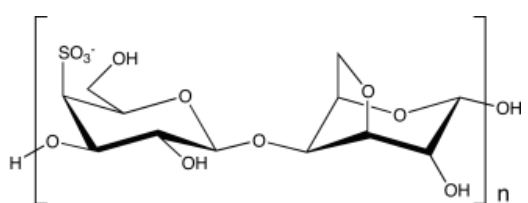

(b)

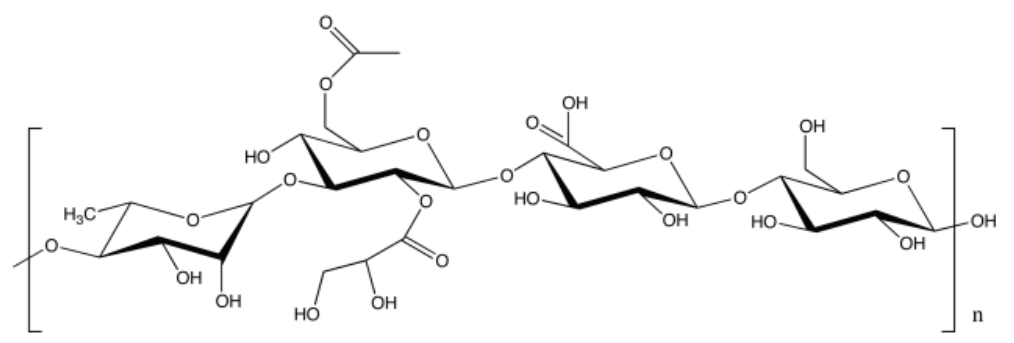

(c)

Fig. 1 Polysaccharides used to make highly hydrated gels. (a) agarose, (b) K-carrageenan, and (c) gellan gum

Adsorption of polysaccharides to surfaces is of importance in the mineral industry where they are used in separation processes and in the pharmaceutical industry where specific polysaccharide interactions are important in drug delivery $[10,11]$. The widespread interest in bioadhesion, both in cell interactions and in the behavior of bacteria, has led to many investigations into the interactions between polysaccharides and other materials [12-16]. The interaction of polysaccharides chains with each other and with other materials is dominated by hydrogen-bonding $[17,18]$. Thus on the one hand, they can lead to low friction due to the strong solvation of the chains by water but will also lead to high adhesion when the chains can form hydrogen bonds to, for example, a bioadhesive surface.

\section{Adsorption and adhesion of polysaccharides}

One of the most widely known properties of sugars is that they become sticky when wet. This is due to the hydrogen bonding between the sugar molecules being broken up in the presence of water. As long as too little water is available for complete hydration, then the hydroxyl groups will hydrogen bond to other materials [19], including the skin. Individual hydrogen bonds between hydroxyl groups or between a hydroxyl group and water are generally weak at about 20 to $25 \mathrm{~kJ} / \mathrm{mol}$. [17,18,20-23]. However, a cooperative effect occurs that allows strong interactions to be formed by large numbers of hydrogen bonds [17,24]. An extreme example of this is in cellulose fibrils, where hydrogen bonding occurs along the entire polysaccharide (Fig. 2) [25].

In aqueous solution, hydrogen bonding between the polysaccharide and materials is not static, even when the adsorption of the carbohydrate onto a surface is irreversible. When long polysaccharide chains adsorb onto oxide surfaces, the positions of hydrogen bonding varies between the monosaccharide units ensuring that the chain remains attached to the surface although the individual hydrogen bonds are continuously broken and reformed [21,26].

Polysaccharides are widely used in the mineral industry as mineral depressants to assist is the separation process [11]. Interest in the interactions of polysaccharides with surfaces also arises from their role in the adhesion of bacterial cells [26]. The adsorption properties of polysaccharides are generally thought of as being dominated by hydroxyl-metal ion interactions and hydrogen bonding [11,18,26-28]. The measurement of interactions between cellulose and silica particles has been investigated using atomic force microscopy (AFM) [29]. Desorption forces were measured in aqueous solutions designed to influence the strength of the electrostatic repulsion between the cellulose and silica surface. No evidence of an attractive van der Waals force was found and it was concluded that the adhesion between silica and cellulose was dominated by hydrogen bonding, whereas the repulsion was dominated by electrostatic forces [29].

However, polysaccharides can also display amphiphilic behavior. Many sugars are able to form a conformation in which the axial hydrogens of the

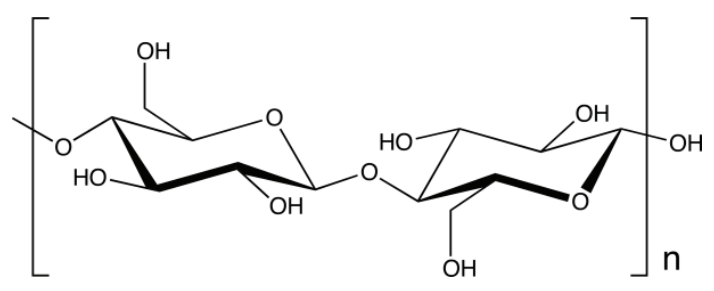

Fig. 2 Repeating unit of cellulose 
methylene groups on one side of the ring form the hydrophobic face (Fig. 3) [30]. The hydrophobicity can be sufficiently strong to stabilize a secondary structure. For example, amylose forms a helix with a hydrophobic inner shell and a hydrophilic outer shell [11]. This hydrophobic property was used to study the lubricating ability of aqueous solutions of polysaccharides between PDMS substrates. Measurements of the dry and wet mass with surface plasmon resonance (SPR) and quartz crystal microbalance with dissipation (QCM-D), respectively, showed significantly different adsorption behavior for different polysaccharides [31].

Not all polysaccharides display a detectable hydrophobicity. Whereas sugars such as dextrin and pectin display behavior associated with hydrophobic interactions, such as solubilizing hydrophobic molecules and adsorbing to hydrophobic surfaces, others such as cellulose and dextran do not [30,31]. This property is considered to be particularly important in the process of recognition at cell surfaces [30].

Polysaccharides that display only hydrophilic behavior can be modified, usually by etherification or esterification, to allow hydrophobic interactions to be formed $[32,33]$.

In the absence of a cooperative effect, hydrogen bonding is weaker than electrostatic and hydrophobic interactions in aqueous solution, thus, hydrogen bonding will only be the dominant interaction where the other two do not occur. Examples of polysaccharides adsorbing via hydrogen bonding include hyaluronan on mica and dextran on silicon oxides [21,34]. In both cases, the interactions are weak due to repulsive forces that include the solvation energy of the polysaccharide as well as Lewis acid repulsion $[11,35,36]$. Studies show that polysaccharides are not a homogeneous group and can behave very differently depending on the monosaccharide units, the position of glycosidic bond in the carbohydrate ring and the type of monosaccharide $[30,31,37]$.

Other strategies that have been used to facilitate studies on polysaccharides at surfaces include the covalent attachment of a positively charged backbone [38,39], alkali treatment [40], and the chemical

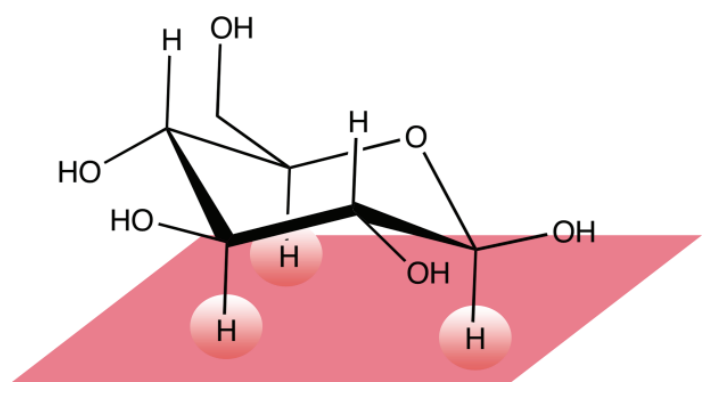

Fig. 3 Diagram of the hydrophobic face of the monosaccharide glucose attachment of the polysaccharide to the surface $[41,42]$.

\section{Friction}

The friction of a variety of surfaces sliding against each other in the presence of carbohydrates has been investigated on all length scales. These studies include systems where the carbohydrate is attached to or adsorbed onto the surface directly, adsorbed via a polymer backbone, as well as experiments where there is no detectable interaction between the surface and the carbohydrate $[2,3,7,9,31,34,41,43-54]$.

Examples of non-adsorbing polysaccharides that have been investigated for their friction properties, using a silicon elastomer and stainless steel as the contacting materials, are xanthan gum and guar gum [51]. Xanthan gum is made up of a glucose backbone with branching at every second glucose. The pentasaccharide repeat units are comprised of glucose, mannose, and glucuronic acid in the molar ratio 2:2:1 [55]. Guar gum is made up of a backbone of mannose units with side chains of galactose at every second mannose [56]. As with dextran and cellulose, neither the xanthan nor the guar gum show significant hydrophobic properties. Measurements were carried out on both the hydrophobic elastomer and elastomer that had been rendered hydrophilic by plasma treatment [51]. When the measurements were carried out with the hydrophobic elastomer, the friction coefficient was higher at low speeds for the polysaccharide solutions than for pure water. When using the hydrophilic surface, the friction coefficients were similar. It was proposed that the increase in friction of steel against the hydrophobic surface was due to the polysaccharide becoming trapped at the inlet to the contact. Thus, in the absence of significant adsorption onto the surface, the polysaccharides does not reduce friction in the boundary regime [51].

Polysaccharides that do form hydrophobic faces, such as pectin and locust bean gum do adsorb onto hydrophobic surfaces from aqueous solution and influence friction in the boundary and mixed regimes [31]. Pectin, a group of polysaccharides containing galacturonic acid residues [57], for example, adsorbs onto hydrophobic PDMS in large amounts to form a highly hydrated boundary film that has a low friction coefficient when sliding against a counter-surface of PDMS [31]. Locust bean gum, which is made up of a $\beta$-1,4-D-mannopyranose backbone with single unit side chains of $\alpha-1,6-\mathrm{D}$-galactopyranose residues [57], also adsorbs onto the PDMS but to a much lesser extent and forms a more rigid film resulting in a higher friction coefficient [31]. The ability of a polysaccharide to adsorb onto hydrophobic surfaces is an indication of its ability to form a hydrophobic face on the carbohydrate residues. However, that low friction is observed when sliding against a surface with the same chemistry, to which the polysaccharide could adsorb equally strongly is surprising. This may be due to the formation of a 
conformation of at the surface of the adsorbed polysaccharide layer that does not allow strong hydrophobic interactions to be formed.

Hydrogen bonding is generally weaker than electrostatic and hydrophobic interactions in aqueous solution, thus, hydrogen bonding will only be the dominant interaction where the other two do not occur. The exceptions to this are when hydrogen bonding occurs over long distances such as in cellulose or a high density of hydrogen bonding is present as in lectin and lectin-type binding [24]. An example of the use of hydrogen bonding to effect adsorption is provided by the adsorption of a negatively charged polysaccharide derived from the red alga Porphyridium sp. [7] onto negatively charged mica. This polysaccharide is made up of xylose $(38 \%)$, galactose $(24 \%)$, glucose $(22 \%)$, and glucuronic acid units $(10 \%)$ as well as minor quantities of other monosaccharides [7]. The atomic force imaging in this study is particularly interesting as the significant changes in structure that can occur when the polysaccharide dries out are demonstrated. In the fully hydrated form, the Porphyridium sp. polysaccharide adsorbs as small globular domains to form a monolayer. After rinsing the substrate and drying, microfibrils of the polysaccharide are observed that are several micrometers long [7]. As the hydroxyl groups on the carbohydrate become less hydrated, the amount of intermolecular hydrogen bonding will increase forming the observed structures. Thus images of adsorbed polysaccharides in the dry form will not necessarily provide information on how it will behave in aqueous solution.

The adhesion and friction properties of the polysaccharide monolayer in aqueous solution was investigated using SFA. Although the Porphyridium $s p$. polysaccharide adsorbed onto the mica, no bridging between the opposing mica surfaces were detected [7]. This is consistent with the observation that the adsorbed polysaccharide could easily be moved over the surface with the AFM tip. When both surfaces were coated with polysaccharide and force applied, then an adhesion between the surfaces was observed and attributed to hydrogen bonding between polysaccharide chains [7,37]. At low applied load, up to $40 \mathrm{mN}$, a friction coefficient of 0.015 was observed, similar to the values observed at low loads in macroscopic ball on disc measurements [5,7]. For a ceramic silicon nitride ball sliding against alumina, a sharp increase in friction was observed at circa $0.8 \mathrm{~N}$. In the SFA, and a shear-induced increase in adhesion was observed [7]. Another negatively charged polysaccharide, hyaluronan, was squeezed out of the contact in the SFA, showing weaker hydrogen-bonding to the surface than the Porphyridium sp. polysaccharide [7,34].

When both substrates at the sliding contact are the same then the adsorption of the polysaccharide onto one surface can be expected to result in an adhesive force at the other surface. That this does not necessarily lead to a high friction coefficient can be seen in the results of the SFA experiments by Gourdon et. al., which may be due to

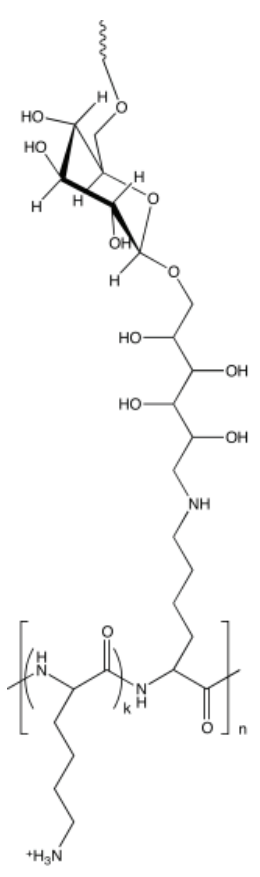

(a)

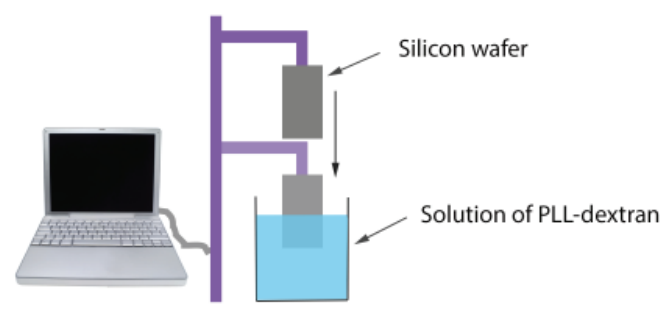

(b)

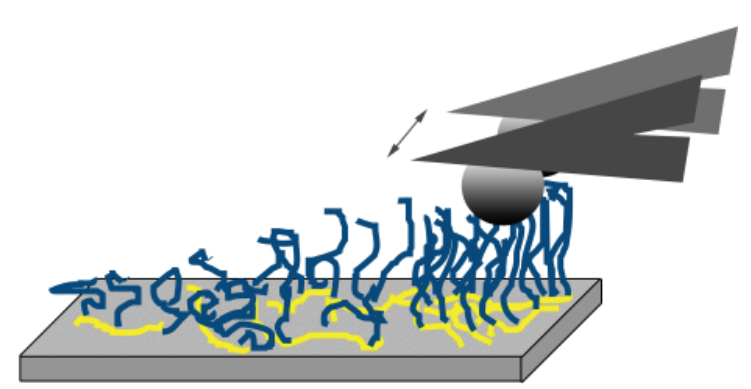

(c)

Fig. 4 (a) Structure PLL-dextran showing attachment of the lysine amino group to the end of the dextran chain, (b) gradients were generated by dipping the silicon substrate into a solution of PLL-dextran at a rate determined by previously performed adsorption measurements, (c) friction was measured along the gradient using colloidal probe AFM 
the low strength of the individual hydrogen bonds [7]. Another strategy that has been used to adsorb polysaccharides onto surfaces is to attach the carbohydrate to a charged backbone, resulting in an asymmetric coverage of the substrate $[38,54]$. Dextran has been attached to poly L-lysine (PLL) at the reducing end via reductive amination (Fig. 4a) [38]. The resulting brush-like polymer adsorbs onto the surface via the unreacted, positively charged lysine residues. The electrostatic interaction between the amino groups of the lysine and the silicon oxide surface are stronger than the hydrogen bonding between the dextran and silicon oxide $[15,16,21,22,58,59]$. Therefore, any dextran that adsorbs on the surface will quickly be replaced by poly-L lysine and the adsorbed polymer brush will have a conformation in which the charged backbone interacts with the surface while the polysaccharide chain extends into the aqueous solution.

The friction behavior of PLL-dextran has been investigated on the nano and macroscale $[38,49]$. On the macroscale the friction coefficient was found to be higher at all brush densities for the dextran than for another polymer brush, PLL-PEG [38]. The friction behavior was studied in more detail using AFM, in which a borosilicate colloidal probe slid against a gradient of densities of PLL-dextran on a silicon substrate (Fig. 4) [49]. The dextran used in these experiments had a molecular weight of $5 \mathrm{kDa}$. The gradients were produced by dipping the silicon substrate into a solution of the PLL-dextran at a rate that gave a range of brush densities of $L / 2 R_{\mathrm{g}}=4$ to 0.5 , where $L$ is the distance between dextran chains and $R_{\mathrm{g}}$ is the radius of gyration [49].

At lower loads, friction measurements of both a bare colloidal probe and a probe coated with PLL-dextran sliding against the gradient showed the expected decrease in friction coefficient with increasing brush density (Fig. 5). At each brush density, the friction coefficient with the bare borosilicate probe was always higher than for the coated probe [49]. This is consistent with the behavior that has been observed for other brush-like systems, such as PLL-PEG, in which osmotic pressure and entropy are believed to play a large role in maintaining a low friction aqueous layer between the sliding surfaces [60-63].

For each brush density measured, the friction coefficient increased at some load, referred to as the transition load, which increased with increasing brush density [49]. At loads higher than the transition loads, the friction coefficient increased to very high values, above 1 , that indicated that the surfaces were sticking together (Fig. 5).

Contrary to the low-load friction, at high loads the friction coefficient was lower for the bare colloidal probe sliding against PLL-dextran at each brush density than for the probe coated with PLL-dextran. Additionally, the friction coefficient increased with increasing brush density [49]. The very high friction was attributed to a decrease in hydration and an increase in hydrogen bonding between the contacting surfaces (Fig. 6). In the case of the bare probe, the hydrogen bonding to dextran in the fully hydrated state is weak [21,26], but on dehydration, the number of hydrogen bonds between the surface and polysaccharide will increase as the repulsion caused by solvation of the dextran decreases. Studies on the adsorption of starch onto silicon oxide have demonstrate that solvation energy, as well as Lewis acid repulsive forces, play a role in reducing hydrogen bonding between polysaccharide and the oxide surface

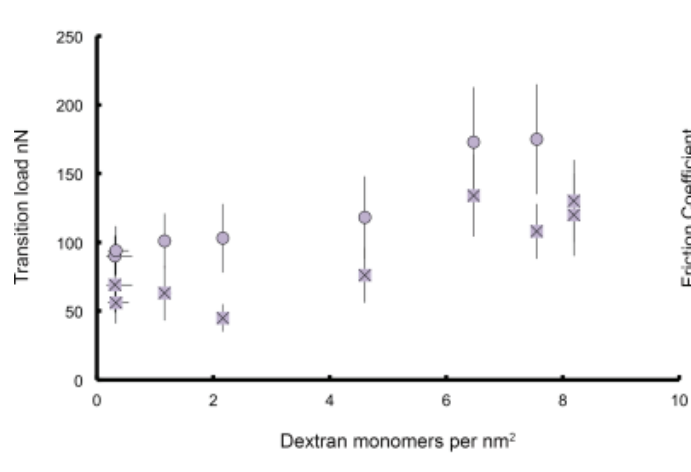

(a)

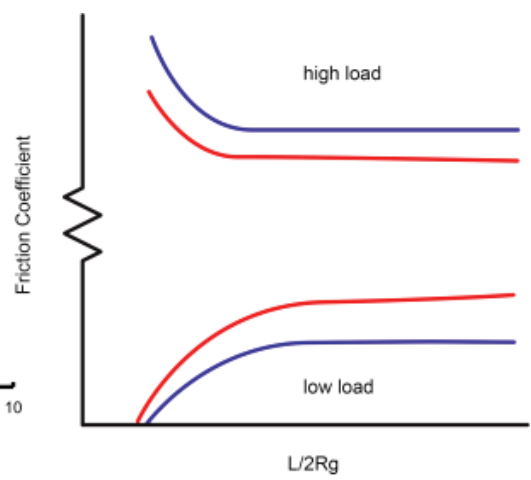

(b)

Fig. 5 (a) At each brush density measured, the friction coefficient showed a sharp increase at some load. This load at which the friction coefficient increased was described as the transition load. The graph shows the transition load against the number of dextran monomers per $\mathrm{nm}^{2}$ adsorbed onto the surface for a bare colloidal probe sliding against PLL-dextran (squares) and a PLL-dextran coated probe sliding against PLL-dextran (circles). (b) The friction coefficients are mirrored in the low and high load region for the bare colloidal probe sliding against PLL-dextran (red line) and a PLL-dextran coated probe sliding against PLL-dextran (blue line) along the PLL-dextran gradient 
[21,35]. The hydrogen bonding of polysaccharides to oxide surfaces is reversible, as seen in the many examples of bioadhesion [1,21,64], and thus removing the load results in rehydration of the dextran. It can be expected that the repulsive forces between the dextran chains [36] and between dextran and silicon oxide [21] will encourage rehydration when the load is removed. As with cellulose [29], attractive van der Waals forces are negligible for dextran [21]. However, unlike cellulose, dextran does not form highly ordered hydrogen bonding over long ranges. A phenomenon in cellulose that results in the poor dissolution behavior of the polysaccharide. When compared to the friction measurements on the polysaccharide from Porphyridium $s p$., it can be concluded that the stronger electrostatic repulsions in the case of the negatively charged polysaccharide on mica than for dextran against silicon oxide prevent to formation of a strong, adhesive hydrogen bonding at high loads [7].

A similar situation applies to the interaction of the two dextran coated surfaces (Fig. 7). As water is forced out of the contact, the number of hydrogen bonds between hydroxyl groups on the polysaccharide will increasingly exceed the repulsion between dextran chains [36]. The adhesion caused by this increase in hydrogen bonding will be higher for two chains in contact than for the polysaccharide and silicon oxide

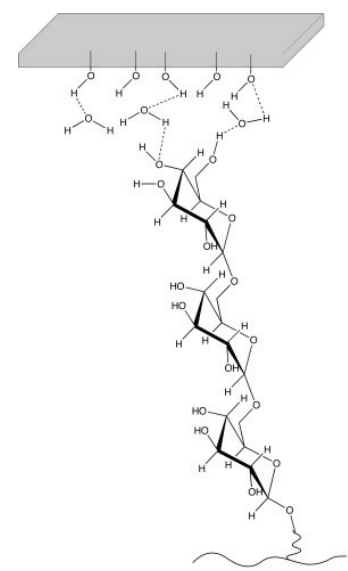

(a)

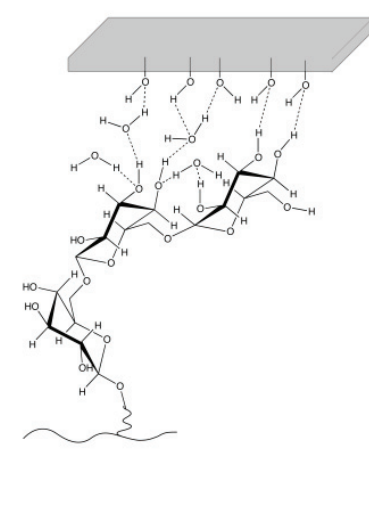

(b)
Fig. 6 (a) At low load, there will be few contact points between the dextran chains and the silicon oxide surface. The osmotic pressure in the brushes will add to the repulsive forces resulting from Lewis acid interactions and solvation, reducing the probability of hydrogen bonds forming. (b) At high load, the area of contact between the polysaccharide chain and surface increases, increasing the number of possible hydrogen bonding sites to the point where this is sufficient to overcome the repulsive forces surface due to the larger number of possible contact points per unit area[17,18]. Smaller dextran chains are not easily dissolved following aggregation through hydrogen bonding [65-67]. However, the reversibility of the friction coefficients following sliding of dextran against dextran suggests that the polysaccharide has not become sufficiently concentrated to cause such aggregation [65-67]. As well as dehydration, the formation of aggregates resistant to dissolution also requires orderly molecular association as shown by $\mathrm{x}$-ray analysis [67]. A high degree of order is improbable in the chains interacting with each other during sliding at high loads.

The behavior of the dextran brushes in these two regimes of low and high friction can be compared to the friction and adhesive properties of mucins $[16,50,68]$. Mucins have a structure that allows the protein backbone to adsorb on the surface and the oligosaccharide or polysaccharide moieties to extend into the aqueous solution in a brush-like conformation [2,3].

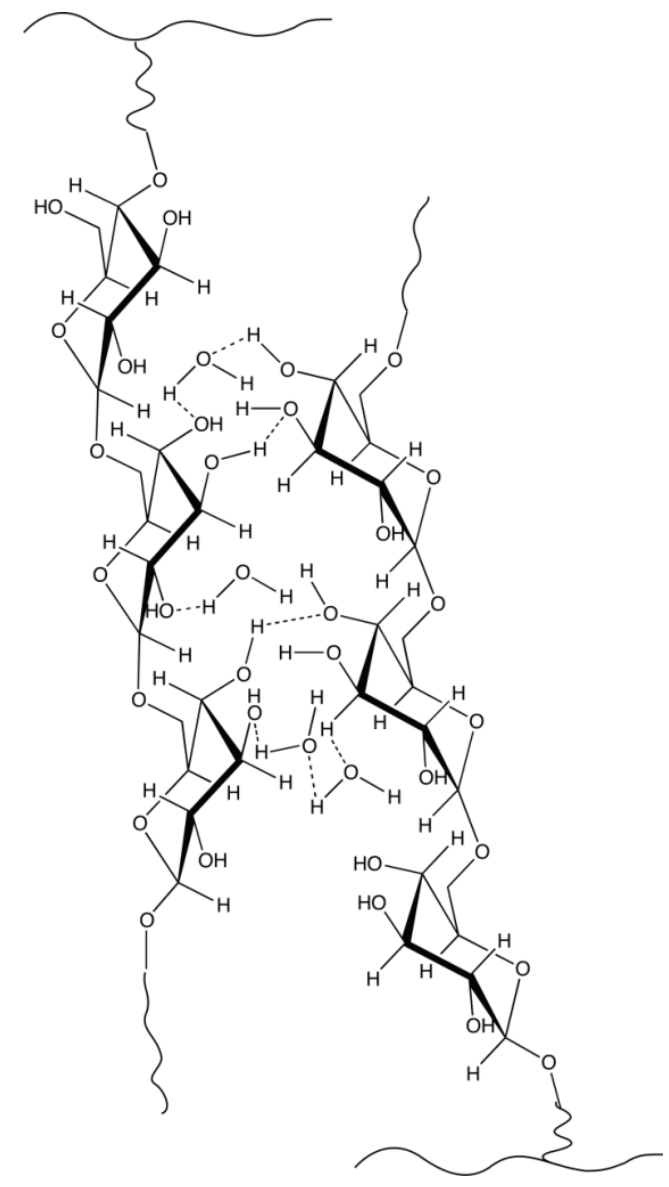

Fig. 7 Hydrogen bonding between intertwined polysaccharides chains adsorbed onto silicon oxide can occur either directly between polysaccharide hydroxyl groups of through intermediate water molecules 
SFA measurements on lubricin adsorbed onto mica, for example, show a transition in friction coefficient from low to high values at a load of approximately $0.4 \mathrm{mN}$. The squeezing out of water in the AFM experiments at high load can be compared to the dehydration mechanism of mucoadhesion [69]. The strength of hydrogen bonds vary a great deal $[10,20]$, but they are weaker than electrostatic and covalent bonds. Therefore, in order for hydrogen bonding to become a significant component of adhesion a large number of hydrogen bonds are required. This can be compared to the use of weak van der Waals forces in the adhesion mechanism of geckos feet to hydrophobic surfaces [70]. The real contact area at the feet of the gecko is sufficiently high to make adhesion feasible even when only very weak London forces are possible [70]. In mucoadhesion, the number of contacting points at which hydrogen bonding can occur is increased by mixing of the mucoadhesive and the mucus $[16,59,69,71]$. Dehydration of the mucus is achieved by using a mucoadhesive that has a higher affinity for water than the mucus and adhesive failure occurs when there is overhydration of the system $[16,59,69,71]$.

In order to determine the influence of the brush structure of the polysaccharide on the low friction at low load and high adhesion at high load, large dextran chains were attached covalently to the surface [72]. Poly(allylamine)-graft-perfluorophenylazide (PFPA) was adsorbed onto the silicon surface from aqueous solution through the positively charged amine. Dextran with a molecular weight of $2 \mathrm{MDa}$ was spin-coated onto this and covalent bonding between the two polymers was achieved with UV irradiation. This eliminates nitrogen from the azide groups on PFPA leaving highly reactive nitrenes, which insert into any bond in the vicinity. Gradients were produced by exposing the surface to UV for differing times along the substrate.

At low dextran coverage, the friction coefficient was high even at low loads. At higher coverage, a transition load was observed as with the brush-like PLL-dextran [72]. However, a comparison of the two systems showed that a higher coverage of the random PFPA-dextran than the brush PLL-dextran in terms of monomers $/ \mathrm{nm}^{2}$ was required to achieve the same transition load (Fig. 8). That is, fewer monomers are required in the brush-like structure to maintain the lower friction regime. Additionally, the differences between the friction in the low and high load regimes were not as large when the large dextran was attached randomly to the surface as in the case of the brush-like structure [72]. The lower friction coefficients observed for PFPA-dextran above the transition load suggest that the randomly attached polymer was not able to form a conformation that allowed as many hydrogen bonds to be formed as in the case of the PLL-dextran brush. Thus, from these experiments it would appear that the brush-like structure of mucins is optimized for both low friction and high adhesion depending on the degree of hydration and the

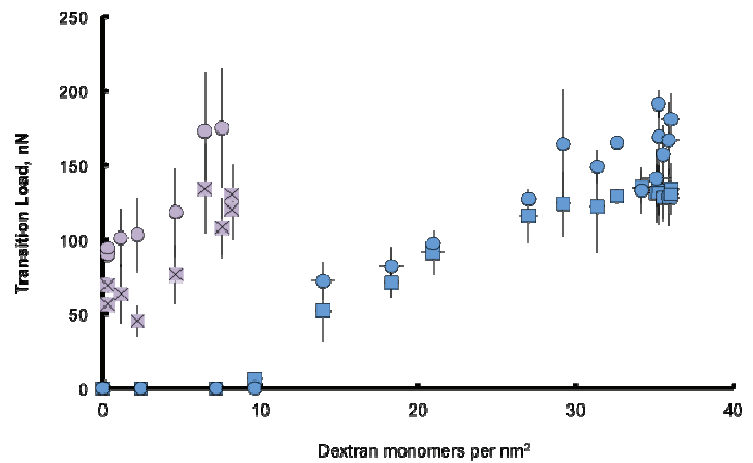

Fig. 8 Comparison of the transition loads for PLL-dextran (purple) with those for PFPA-dextran (blue) at different adsorbed mass for asymmetric contacts of bare probe against dextran (squares) and a PLL-dextran coated probe sliding against the dextran gradient (circles)

applied load [16,53,69,73-75].

\section{Conclusions}

Providing the interactions between polysaccharide and the surface are strong enough to allow adsorption, then low frictions can be achieved due to the ability of the carbohydrate to main a hydrated film at the surface. For neutral polysaccharides, adsorption from aqueous solution occurs through hydrogen-bonding or hydrophobic interactions, whereas the charged polysaccharides can also adsorb via electrostatic interactions depending on the chemistry of the surface. The ability to maintain low friction with increasing load requires that the interaction with the surface is strong enough to prevent the polysaccharide from being squeezed out of the contact. However, when the interaction with the surface becomes too strong, then high adhesion and high friction coefficients will be observed. Repulsion between oxide surfaces and polysaccharides is dominated by Lewis acid interactions and the solvation energy. For hydrogen bonding between the surface and polysaccharide to be effective, the number of hydrogen bonds formed has to be sufficient to overcome the repulsion. Therefore, with a low contact area between the polysaccharide and surface, interactions will be weak. As water is squeezed out of the contact, repulsion due to solvation will decrease and, additionally, the area of contact between polysaccharide chains as well as between polysaccharide and surface will increase, thus enhancing hydrogen bonding to the point that very high friction coefficients are observed. This adhesion is reversed when the polysaccharide chains are rehydrated as the load is removed. 


\section{References}

[1] Graham, L. D., "Biological Adhesives from Nature," Encyclopedia of Biomaterials and Biomedical Engineering, Informa Healthcare, New York, 2008, 236-253.

[2] Lee, S., "Sticky and Slippery: Interfacial Forces of Mucin and Mucus Gels," Aqueous Lubrication, World Scientific Publishing Co., 2013, 1-40.

[3] Lee, S. and Spencer, N. D., "Sweet, Hairy, Soft, and Slippery," Science, 319, 2008, 575-576.

[4] Li, J., Liu, Y., Luo, J., Liu, P. and Zhang, C., "Excellent Lubricating Behavior of Brasenia Schreberi Mucilage,” Langmuir, 28, 2012, 7797-7802.

[5] Arad, S. M., Rapoport, L., Moshkovich, A., van Moppes, D., Karpasas, M., Golan, R. and Golan, Y., "Superior Biolubricant from a Species of Red Microalga," Langmuir, 22, 2006, 7313-7317.

[6] Gasljevic, K., Hall, K., Chapman, D. and Matthys, E.F., "Drag-Reducing Polysaccharides from Marine Microalgae: Species Productivity and Drag Reduction Effectiveness," J. Appl. Phycol., 20, 2008, 299-310.

[7] Gourdon, D., Lin, Q., Oroudjev, E., Hansma, H., Golan, Y., Arad, S. and Israelachvili, J., "Adhesion and Stable Low Friction Provided by a Subnanometer-Thick Monolayer of a Natural Polysaccharide," Langmuir, 24, 2008, 1534-1540.

[8] Cann, P. M., "The "Leaves on the Line" Problem - a Study of Leaf Residue Film Formation and Lubricity under Laboratory Test Conditions," Tribol. Lett., 24, 2006, 151-158.

[9] Gong, J. P., Iwasaki, Y. and Osada, Y., "Friction of Gels. 5. Negative Load Dependence of Polysaccharide Gels," J. Phys. Chem. B, 104, 2000, 3423-3428.

[10] Boobbyer, D. N., Goodford, P. J., McWhinnie, P. M. and Wade, R. C., "New Hydrogen-Bond Potentials for Use in Determining Energetically Favorable Binding Sites on Molecules of Known Structure.," J. Med. Chem., 32, 1989, 1083-1094.

[11] Liu, Q., Zhang, Y. H. and Laskowski, J. S., "The Adsorption of Polysaccharides onto Mineral Surfaces: an Acid/Base Interaction," Int. J. Miner. Process., 60, 2000, 229-245.

[12] Berry, M., McMaster, T. J., Corfield, A. P. and Miles, M. J., "Exploring the Molecular Adhesion of Ocular Mucins," Biomacromolecules, 2, 2001, 498-503.

[13] Fukuda, M., "Roles of Mucin-Type O-Glycans in Cell Adhesion.," Biochim. Biophys. Acta., 1573, 2002, 394-405.

[14] Hassan, E. E. and Gallo, J. M., "A Simple Rheological Method for the Invitro Assessment of Mucin-Polymer Bioadhesive Bond Strength," Pharm. Res., 7, 1990, 491-495.
[15] Mahrag Tur, K. and Ch'ng, H.-S., "Evaluation of Possible Mechanism (s) of Bioadhesion," Int. J. Pharm., 160, 1998, 61-74.

[16] Smart, J. D., "The Basics and Underlying Mechanisms of Mucoadhesion," Adv. Drug Deliv. Rev., 57, 2005, 1556-1568.

[17] Prins, L. J., Reinhoudt, D. N. and Timmerman, P., "Noncovalent Synthesis Using Hydrogen Bonding," Angew. Chem. Int. Ed., 40, 2001, 2382-2426.

[18] Zhang, Q., Jaroniec, J., Lee, G. and Marszalek, P. E., "Direct Detection of Inter-residue Hydrogen Bonds in Polysaccharides by Single-Molecule Force Spectroscopy," Angew. Chem. Int. Ed., 44, 2005, 2723-2727.

[19] Chen, T.-S. and Joslyn, M. A., "The Effect of Sugars on Viscosity of Pectin Solutions: II. Comparison of Dextrose, Maltose, and Dextrins," J. Colloid Interf. Sci., 25, 1967, 346-352.

[20] Emsley, J., "Very Strong Hydrogen Bonding," Chem. Soc. Rev., 9, 1980, 91-124.

[21] Jucker, B. A., Harms, H., Hug, S. J. and Zehnder, A J. B., "Adsorption of Bacterial Surface Polysaccharides on Mineral Oxides is Mediated by Hydrogen Bonds," Colloid Surface B, 9, 1997, 331-343.

[22] Lee, "Adhesion and Surface-Hydrogen-Bond Components for Polymers and Biomaterials," J. Adhesion, 67, 1998, $1-18$.

[23] Waite, J. H., "Adhesion in Byssally Attached Bivalves," Biol. Rev., 58, 1983, 209-231.

[24] Lee, Y. C. and Lee, R. T., "Carbohydrate-Protein Interactions - Basis of Glycobiology," Acc. Chem. Res., 28, 1995, 321-327.

[25] Tingaut, P., Zimmermann, T. and Sèbe, G., "Cellulose Nanocrystals and Microfibrillated Cellulose as Building Blocks for the Design of Hierarchical Functional Materials," J. Mater. Chem., 22, 2012, 20105-20111.

[26] Jucker, B. A., Harms, H. and Zehnder, A. J., "Polymer Interactions between Five Gram-Negative Bacteria and Glass Investigated using LPS Micelles and Vesicles as Model Systems," Colloid Surface B, 11, 1998, 33-45.

[27] Bechtold, T., Manian, A. P., Öztürk, H. B., Paul, U. Široká, B., Široký, J., Soliman, H., Vo, L. T. T. and Vu-Manh, H., "Ion-Interactions as Driving Force in Polysaccharide Assembly," Carbohyd. Polym., 93, 2013, 316-323.

[28] Taylor, L. S. and Zografi, G., "Sugar-Polymer Hydrogen Bond Interactions in Lyophilized Amorphous Mixtures," J. Pharm. Sci., 87, 1998, 1615-1621.

[29] Radtchenko, I. L., Papastavrou, G. and Borkovec, M., "Direct Force Measurements between Cellulose Surfaces and Colloidal Silica Particles," Biomacromolecules, 6, 2005, 3057-3066. 
[30] Balasubramanian, D., Raman, B. and Sundari, C. S., "Polysaccharides as Amphiphiles," J. Am. Chem. Soc., 115, 1993, 74-77.

[31] Stokes, J. R., Macakova, L., Chojnicka-Paszun, A., de Kruif, C. G. and de Jongh, H. H. J., "Lubrication, Adsorption, and Rheology of Aqueous Polysaccharide Solutions," Langmuir, 27, 2011, 3474-3484.

[32] Fournier, C., Leonard, M., Le Coq-Leonard, I. and Dellacherie, E., "Coating Polystyrene Particles by Adsorption of Hydrophobically-Modified Dextran," Langmuir, 11, 1995, 2344-2347.

[33] Rouzes, C., Durand, A., Leonard, M. and Dellacherie, E., "Surface Activity and Emulsification Properties of Hydrophobically Modified Dextrans," J. Colloid. Interf. Sci., 253, 2002, 217-223.

[34] Tadmor, R., Chen, N. and Israelachvili, J., "Normal and Shear Forces between Mica and Model Membrane Surfaces with Adsorbed Hyaluronan," Macromolecules, 36, 2003, 9519-9526.

[35] Ma, X., "Role of Solvation Energy in Starch Adsorption on Oxide Surfaces," Colloid Surface A, 320, 2008, 36-42.

[36] van Oss, C. J., "Long-Range and Short-Range Mechanisms of Hydrophobic Attraction and Hydrophilic Repulsion in Specific and Aspecific Interactions," J. Mol. Recognit., 16, 2003, 177-190.

[37] Sundaralingam, M., "Some Aspects of Stereochemistry and Hydrogen Bonding of Carbohydrates Related to Polysaccharide Conformations," Biopolymers, 6, 1968, 189-213.

[38] Perrino, C., Lee, S. and Spencer, N. D., "End-Grafted Sugar Chains as Aqueous Lubricant Additives: Synthesis and Macrotribological Tests of Poly(L-Lysine)-graft-Dextran (PLL-g-Dex) Copolymers," Tribol. Lett., 33, 2009, 83-96.

[39] Espinosa-Marzal, R. M., Nalam, P. C., Bolisetty, S. and Spencer, N. D., "Impact of Solvation on Equilibrium Conformation of Polymer Brushes in Solvent Mixtures," Soft Matter, 9, 2013, 4045-4057.

[40] Kawaguchi, T. and Hasegawa, M., "Structure of Dextran-Magnetite Complex: Relation between Conformation of Dextran Chains Covering Core and Its Molecular Weight," J. Mater. Sci.-Mater. M, 11, 2000, 31-35.

[41] Benz, M., Chen, N. and Israelachvili, J., "Lubrication and Wear Properties of Grafted Polyelectrolytes, Hyaluronan and Hylan, Measured in the Surface Forces Apparatus," J. Biomed. Mater. Res. A, 71A, 2004, 6-15.

[42] Martwiset, S., Koh, A. E. and Chen, W., "Nonfouling Characteristics of Dextran-Containing Surfaces," Langmuir, 22, 2006, 8192-8196.

[43] Bernadsky, G. and Rosenberg, E., "Drag-Reducing Properties of Bacteria from the Skin Mucus of the
Cornetfish (Fistularia Commersonii)." Microb. Ecol., 24, 1992, 63-76.

[44] Chang, D. P., Abu-Lail, N. I., Coles, J. M., Guilak, F., Jay, G. D. and Zauscher, S., "Friction Force Microscopy of Lubricin and Hyaluronic Acid between Hydrophobic and Hydrophilic Surfaces," Soft Matter, 5, 2009, 3438-3445.

[45] Goren, T., Crockett, R. and Spencer, N. D., "Influence of Solutes on Hydration and Lubricity of Dextran Brushes," CHIMIA, 66, 2012, 192-195.

[46] McNamee, C. E., Yamamoto, S., Kappl, M., Butt, H.-J., Higashitani, K., Dedinaite, A. and Claesson, P. M., "Surface and Friction Forces between Grafted Polysaccharide Layers in the Absence and Presence of Surfactant,” J. Colloid. Interf. Sci., 364, 2011, 351-358.

[47] Misra, R., Li, J., Cannon, G. C. and Morgan, S. E., "Nanoscale Reduction in Surface Friction of Polymer Surfaces Modified with Sc3 Hydrophobin from Schizophyllum Commune," Biomacromolecules, 7, 2006, 1463-1470.

[48] Pettersson, T. and Dedinaite, A., "Normal and Friction Forces between Mucin and Mucin-Chitosan Layers in Absence and Presence of SDS," J. Colloid. Interf. Sci., 324, 2008, 246-256.

[49] Rosenberg, K. J., Goren, T., Crockett, R. and Spencer, N. D., "Load-Induced Transitions in the Lubricity of Adsorbed Poly(L-Lysine)- g-Dextran as a Function of Polysaccharide Chain Density," ACS Appl. Mater. Interfaces, 3, 2011, 3020-3025.

[50] Zappone, B., Ruths, M., Greene, G. W., Jay, G. D. and Israelachvili, J. N., "Adsorption, Lubrication, and Wear of Lubricin on Model Surfaces: Polymer Brush-Like Behavior of a Glycoprotein," Biophys. J., 92, 2007, 1693-1708.

[51] de Vicente, J., Stokes, J. R. and Spikes, H. A., "Lubrication Properties of Non-Adsorbing Polymer Solutions in Soft Elastohydrodynamic (EHD) Contacts," Tribol. Int., 38, 2005, 515-526.

[52] Roba, M., Naka, M., Gautier, E., Spencer, N. D. and Crockett, R., "The Adsorption and Lubrication Behavior of Synovial Fluid Proteins and Glycoproteins on the Bearing-Surface Materials of Hip Replacements," Biomaterials, 30, 2009, 2072-2078.

[53] Coles, J. M., Chang, D. P. and Zauscher, S., "Molecular Mechanisms of Aqueous Boundary Lubrication by Mucinous Glycoproteins," Curr. Opin. Colloid In., 15, 2010, 406-416.

[54] Nalam, P. C., Ramakrishna, S. N., Espinosa-Marzal, R. M. and Spencer, N. D., "Exploring Lubrication Regimes at the Nanoscale: Nanotribological Characterization of Silica and Polymer Brushes in Viscous Solvents," Langmuir, 29, 2013, 10149-10158.

[55] Garcia-Ochoa, F., Santos, V. E., Casas, J. A. and Gomez, E., "Xanthan Gum: Production, Recovery, 
and Properties," Biotechnol. Adv., 18, 2000, 549-579.

[56] Heyne, E. and Whistler, R. L., "Chemical Composition and Properties of Guar Polysaccharides," J. Am. Chem. Soc., 70, 1948, 2249-2252.

[57] de Jong, S. and van de Velde, F., "Charge Density of Polysaccharide Controls Microstructure and Large Deformation Properties of Mixed Gels," Food Hydrocolloids, 21, 2007, 1172-1187.

[58] Hori, K. and Matsumoto, S., "Bacterial Adhesion: From Mechanism to Control," Biochem. Eng. J., 48, 2010, 424-434.

[59] Smart, J. D., "Drug Delivery using Buccal-Adhesive Systems," Adv. Drug Deliv. Rev., 11, 1993, 253-270.

[60] Lee, S., Iten, R., Muller, M. and Spencer, N. D., "Influence of Molecular Architecture on the Adsorption of Poly(Ethylene Oxide)-Poly(Propylene Oxide)-Poly(Ethylene Oxide) on PDMS Surfaces and Implications for Aqueous Lubrication," Macromolecules, 37, 2004, 8349-8356.

[61] Muller, M., Lee, S., Spikes, H. A. and Spencer, N. D., "The Influence of Molecular Architecture on the Macroscopic Lubrication Properties of the Brush-Like Co-Polyelectrolyte Poly(L-Lysine)-g-Poly(Ethylene Glycol) (PLL-g-PEG) Adsorbed on Oxide surfaces," Tribol. Lett., 15, 2003, 395-405.

[62] Muller, M. T., Yan, X., Lee, S., Perry, S. S. and Spencer, N. D., "Lubrication Properties of a Brushlike Copolymer as a Function of the Amount of Solvent Absorbed Within the Brush," Macromolecules, 38, 2005, 5706-5713.

[63] Drobek, T. and Spencer, N. D., "Nanotribology of Surface-Grafted PEG Layers in an Aqueous Environment," Langmuir, 24, 2008, 1484-1488.

[64] Grabovac, V., Guggi, D. and Bernkop-Schnuerch, A., "Comparison of the Mucoadhesive Properties of Various Polymers," Adv. Drug Deliv. Rev., 57, 2005, 1713-1723.

[65] Aizawa, M., Suzuki, S., Kuoka, T., Nakajima, N. and Iwao, Y., "Stability of Dextran Solutions," Bull. Chem. Soc. Japan, 49, 1976, 2061-2065.
[66] Fedin, E. I., Tsitsishvili, V. G., Grinberg, V. Y., Bakari, T. I. and Tolstoguzov, V. B., "A Study of Dextran Hydration in Dilute, Aqueous Solution by the Proton Magnetic Relaxation Method," Carbohyd. Res., 39, 1975, 193-199.

[67] Jeanes, A., Schieltz, N. C. and Wilham, C. A., "Molecular Association in Dextran and in Branched Amylaceous Carbohydrates," J. Biol. Chem., 176, 1948, 617-627.

[68] Mortazavi, S. A., "An in-Vitro Assessment of Mucus Mucoadhesive Interactions," Int. J. Pharm., 124, 1995, 173-182.

[69] Smart, J. D., "The Role of Water Movement and Polymer Hydration in Mucoadhesion," Bioadhesive Drug Delivery Systems: Fundamentals, Novel Approaches and Development, CRC Press, 1999, 11-23.

[70] Autumn, K., Sitti, M., Liang, Y. A., Peattie, A. M., Hansen, W. R., Sponberg, S., Kenny, T. W., Fearing, R., Israelachvili, J. N. and Full, R. J., "Evidence for Van Der Waals Adhesion in Gecko Setae," Proc. Natl. Acad. Sci. USA., 99, 2002, 12252-12256.

[71] Mortazavi, S. A. and Smart, J. D., "An Investigation into the Role of Water-Movement and Mucus Gel Dehydration in Mucoadhesion," Journal of Controlled Release, 25, 1993, 197-203.

[72] Goren, T., Spencer, N. D. and Crockett, R., "Impact of Chain Morphology on the Lubricity of Surface-Grafted Polysaccharides," RSC Advances, 4, 2014, 21497-21503.

[73] Meloni, M., De Servi, B., Marasco, D. and Del Prete, S., "Molecular Mechanism of Ocular Surface Damage: Application to an in Vitro Dry Eye Model on Human Corneal Epithelium," Mol. Vis., 17, 2011, 113-126.

[74] Mortazavi, S. A. and Smart, J. D., "An in-Vitro Method for Assessing the Duration of Mucoadhesion," J. Control. Release, 31, 1994, 207-212.

[75] Patel, M. M., Smart, J. D., Nevell, T. G., Ewen, R. J., Eaton, P. J. and Tsibouklis, J., "Mucin/Poly(Acrylic Acid) Interactions: a Spectroscopic Investigation of Mucoadhesion," Biomacromolecules, 4, 2003, 1184-1190. 